\title{
Concerning the Calculation of the Color Gamut in a Digital Camera
}

\section{Francisco Martínez-Verdú, ${ }^{1 *}$ M. J. Luque, ${ }^{2}$ P. Capilla, ${ }^{2}$ and J. Pujol ${ }^{3}$}

\author{
${ }^{1}$ Department of Optics, University of Alicante, Apartado de Correos n 99, 03080 Alicante, Spain \\ ${ }^{2}$ Department of Optics, University of Valencia, Dr. Moliner s/n, 46100 Burjassot, Valencia, Spain \\ ${ }^{3}$ Department of Optics and Optometry, Center for Development of Sensors, Instrumentation and Systems (CD6), \\ Technical University of Catalonia, Rambla de Sant Nebridi $n^{\circ}$ 10, 08222 Terrassa, Barcelona, Spain
}

Received 3 January 2004; revised 25 November 2005; accepted 11 January 2006

\begin{abstract}
Several methods to determine the color gamut of any digital camera are shown. Since an input device is additive, its color triangle was obtained from their spectral sensitivities and it was compared with the theoretical sensors of Ives-Abney-Yule and MacAdam. On the other hand, the RGB digital data of the optimal or MacAdam colors were simulated to transform them into XYZ data according to the colorimetric profile of the digital camera. From this, the MacAdam limits associated to the digital camera are compared with the corresponding ones of the CIE-1931 $X Y Z$ standard observer, resulting that our color device has much smaller MacAdam loci than those of the colorimetric standard observer. Taking this into account, we have estimated the reduction of discernible colors by the digital camera applying a chromatic discrimination model and a packing algorithm to obtain color discrimination ellipses. Calculating the relative decrement of distinguishable colors by the digital camera in comparison with the colorimetric standard observer at different luminance factors of the optimal colors, we have found that the camera distinguishes considerably fewer very dark than very light ones, but relatively much more colors with middle lightness ( $\mathrm{Y}$ between 40 and 70, or $\mathrm{L}^{*}$ between 69.5 and 87.0). This behavior is due to the short dynamic range of the digital camera response.@ 2006 Wiley Periodicals, Inc. Col Res Appl, 31, 000-000, 2006; Published online in Wiley InterScience (www.interscience. wiley.com). DOI 10.1002/col.00000
\end{abstract}

Key words:digital camera; color triangle; MacAdam limits; color discrimination ellipses

Correspondence to: Francisco martínez-verdú (e-mail: verdu@ua.es)

Contract grant sponsor: Ministerio de Educación y Ciencia (Spain), Contract grant number: DPI2002-00118, DPI2005-08999-C02.

\section{INTRODUCTION}

Color devices ${ }^{1-7}$ are basically divided into input or capture devices (scanners and digital cameras) and output devices (softcopy, such as displays, and hardcopy, such as printers). Scanners, digital cameras, and displays (CRT, LCD/TFT, plasma, etc.) are additive color devices. However, most printing devices ${ }^{8}$ (inkjet, electro-photography, offset, etc.) perform by additive and subtractive color mixing. Successful color management ${ }^{5-11}$ depends on knowing the color gamut and the color profile of the device used. Determining the gamut of output devices (displays, projectors, and printers) is relatively easy, both when colors in display or in paper are generated systematically ${ }^{12}$ and when color profiles are applied. ${ }^{13}$ However, the conceptual problems inherent to determining the gamut of input devices (scanners and digital cameras) are numerous. Displays, projectors, and printers are electro-optical devices, that is, they generate color digital images by physical and electronic procedures that are finally seen in a medium (display, screen, or paper), and so each $R G B$ or CMYK digital data triad corresponds to a single color-stimulus. Scanners and digital cameras are optoelectronic devices, ${ }^{14-16}$ that is, they encode and generate a digital image from the light projecting over them from the original image by means of physical and electronic procedures. Then, this digital image is seen on display and saved in some image file format.

The key factor in the performance of input devices is the univariance principle: spectrally different color stimuli may give rise to identical $R G B$ digital data. Therefore, it is very difficult to determine what color-stimulus corresponds to a $R G B$ triad if the captured scene is not previously known. If we capture a reference scene of known colors (taken from, for example, a color atlas such as Munsell's or the NCS) and 
determine the corresponding $R G B$ values, the next step is to transform the $R G B$ digital data into $X Y Z$ data to determine how the input device encodes these color-stimuli in comparison with the human eye. To do this, we must apply the input-device's color profile to the $R G B$ values to derive the corresponding $X Y Z$ values. Therefore, the color gamut of an input device depends on a color transform ("input device plus transform" gamut). So, it is not clear a priori if any type of color transform might be associated with the "input device alone" gamut, or if it can be calculated by means of other alternative procedures. Note that the color gamut of output devices can be obtained far more simply.

In principle, this work would not be necessary if all input devices were completely linear in an optoelectronic manner and satisfied the Luther condition, ${ }^{17,18}$ that is, if their colormatching functions or scaled spectral sensitivities were exact linear combinations of the color-matching functions of the CIE standard observer. Let $\mathbf{T}_{R G B}$ and $\mathbf{T}_{X Y Z}$ be the color-matching functions of an input device and the colorimetric standard observer, respectively, with 41 rows (from 380 to $780 \mathrm{~nm}$ at $10 \mathrm{~nm}$ step) and 3 columns. The nonfulfillment of Luther condition implies that there is a $3 \times 41$ nonzero matrix $\mathbf{C}$ that links both capture systems as follows:

$$
\mathbf{T}_{X Y Z}{ }^{t}=\mathbf{M} \cdot \mathbf{T}_{R G B}{ }^{t}+\mathbf{C}
$$

where $\mathrm{M}$ is the basic color profile or connection matrix relating both color spaces, ${ }^{19-21}$ which is one of the basic components of any characterization model for these color devices. $\left(\mathbf{A}^{\mathrm{T}}\right.$ is transpose matrix of matrix $\left.\mathbf{A}\right)$.

If matrix $\mathbf{C}$ (or Luther bias) were zero, the $X Y Z$ tristimulus values derived from the color profile would coincide with the real values and the input device would work as a colorimeter, and colors metameric for the eye would also be so for the camera and vice versa. But, in fact, matrix $\mathbf{C}$ is not zero, so this initial error is dragged down all of the complete color profile (from capture to image editing) and finally it is mixed with the reproduction errors caused by the optoelectronic limitation of the dynamic response range, that is, the nonlinear errors associated to response clipping due to noise and saturation. Consequently, due to the nonideal optoelectronic performance and the nonfulfillment of the Luther condition, the input devices, in raw performance (without color rendering to standard output-referred representations), will always show a color gamut different from that of the colorimetric standard observer.

The gamut of the camera can be determined directly by measuring (or predicting) its optimal colors, that is, by determining in each direction of color space the highest colorfulness the stimulus can attain without saturating the camera response. Alternatively, as we do in this article, the camera may be characterized by the way it responds to a given set of reference colors. While in the first case, one is directly controlling the tristimulus values (or their transformations to any colorimetric space) of the stimulus; in the second, one needs to represent in the same space the tristimulus values of the reference stimulus and the camera response. Therefore, the second method can be used only if the camera response can be translated to the same space as the reference stimulus by means of a model. For these reasons, the gamut obtained with the first procedure may be called "input device alone gamut," whilst the second is rather an "input device plus transform gamut."

Two different approaches will be carried out to understand better this subject using a real digital camera. In the first place, if an input device is additive, it is possible to plot into a chromaticity diagram the color triangle associated to this device and to compare it with the theoretical color triangles of sensors that fulfill Luther's condition (IvesAbney-Yule, (Ref. 1, p 128), MacAdam ${ }^{22}$ ). $R G B$ triangles are usually used to represent graphically the color gamut of displays, but we can use them likewise with input devices because they also are additive. Therefore, this approach, that disregards the color profile of the digital camera as it will be seen below, is the simplest calculation to understand initially the "camera alone" gamut of the input device.

The second approach to this subject, based on determining the "camera plus transform" gamut using a color profile, is to select previously the color-stimuli in the scene, either real (Munsell or NCS chips, IT8 or ColorCheckerDC charts, etc.) or simulated (vector decomposition, ${ }^{19,23,24}$ optimal or MacAdam colors, ${ }^{25-27}$ etc.). This implies in turn that the illuminance level, the chromaticity of the light source and the source/detector geometry, are initially fixed. To simulate color capture in these initial conditions, we must use the spectral power distributions of the color-stimuli of the scene to estimate the $R G B$ digital data of the input device. It is assumed that the optoelectronic spectral functions of the input device are known a priori, either by direct measurement or by simulation under some assumptions about the basic performance of the color device. Then, we should finally have a set of colors encoded according to the colorimetric standard observer in any CIE color space, linear (XYZ, $U^{\prime} V^{\prime} W^{\prime}$, etc.) or nonlinear $\left(L^{*} a^{*} b^{*}\right.$, etc.) and the same set of colors encoded by $R G B$ digital data. In the discussion, we address in detail the comparison between this procedure, where the performance of the camera is "translated" in terms of the color space of the human observer, and a previous method ${ }^{28}$ proposed by Morovič and Morovič, where the optimal colors of the camera are computed directly.

Finally, the number of discernible colors of the camera and that of the colorimetric standard observer can be computed by assuming that both have the same color metric. A priori, this is achieved by estimating the number of the discrimination ellipsoids filling the color solid, which in the human case is associated to the MacAdam limits or RöschMacAdam color solid. ${ }^{25-28}$ Usually, the problem is simplified by fixating the luminance factor $Y$ or the lightness $L^{*}$, so the computation of ellipsoids is replaced by the simpler computation of the discrimination ellipses plus the interpolation of the just-noticeable lightness differences between a fixed value and the next one. ${ }^{29-31}$ Experimental data about discrimination ellipses around in the literature ${ }^{32-38}$ are numerous. We have chosen the Krauskopf and Gegenfurtner data $^{37,38}$ because they permit a homogeneous sampling of 
the color solid. This procedure could seem an unnecessary complication of a simple problem, because once the MacAdam loci are computed, we could assume that their areas in a given color space are a measurement of the size of the color gamut of the device (standard observer or camera). However, we will show below that in this way the result obtained would be dependent on the color space used, whereas this would not happen with the new procedure we propose.

Summarizing, the aim of this work is to provide more information about the calculation of the color gamut of a particular input device, a conventional digital camera. Once the experimental data about the colorimetric and spectral characterization are known, we will follow the three steps described above: the color gamut calculation in a chromaticity diagram based on the primaries of the color device ("camera alone" gamut), the color gamut by predicting the response of the camera for MacAdam limits ("camera plus transform" gamut), and the size of the color gamut based on Krauskopf and Gegenfurtner discrimination data.

\section{Methodology, Results, and Discussion}

This section is divided into three reports. As a first approach to the calculation of the color gamut of an input device ("camera alone" gamut), we show a method for obtaining the primary spectra of a digital camera from its colormatching functions, which we will use to plot its color triangle and to compare it with the theoretical primary spectra and color triangles of Ives-Abney-Yule and MacAdam. In the second approach ("camera plus transform" gamut), the capture of optimal colors is simulated to compare the MacAdam limits as encoded by the digital camera under analysis with those of the CIE standard observer. To develop this approach, the camera should be characterized using a model from measured colorimetric data. ${ }^{20,21,39-43}$ Finally, to complete this second approach, we propose a method to estimate and compare the number of colors distinguishable by the digital camera and the CIE standard observer.

The input device used in this work consists of a Sony DXC-930P camera and a Matrox MVP-AT 850 frame grabber, characterized according to a previously developed model. ${ }^{42,43}$

\section{Determination of the Primary Spectra and the Color Triangle of a Digital Camera}

We propose in this section a simple methodology to determine the primary fundamental ${ }^{44}$ spectra $\mathbf{P}_{R G B}$ (according to Cohen's formalism) from the color-matching functions $\mathbf{T}_{R G B}$ when the basic color profile $\mathbf{M}$ is unknown. The mathematical background for this procedure is linear algebra. ${ }^{7,19,20,44}$ According to this formalism, color-matching functions $\mathbf{T}$ (41 rows by 3 columns, from 380 to $780 \mathrm{~nm}$ at $10 \mathrm{~nm}$ steps) and primary spectra $\mathbf{P}$ (41 rows by 3 columns) of any color space associated to additive color reproduction systems (device or human eye) are linked into a dual relationship as follows:

$$
\mathbf{T}^{T} \cdot \mathbf{P}=\mathbf{I}
$$

where $I$ is the $3 \times 3$ identity matrix.

The basic spectral property of displays and projectors is the set of spectra of their primaries. Their color-matching functions can be inferred from these data so that the spectral sensitivities of the input devices can be designed accordingly (Ref. 1, p 478). However, the basic spectral property known a priori (or measured) of the input devices is the set of the spectral sensitivities, but not directly the associated primary spectra. Therefore, if the primary spectra $\mathbf{P}_{R G B}$ of a display or projector are known, we can calculate its colormatching functions. In the same way, if the color-matching functions $\mathbf{T}_{R G B}$ of an input device are known, we can determine the primary fundamental spectra $\mathbf{P}_{R G B}$ as follows:

$$
\mathbf{P}_{R G B}=\mathbf{T}_{R G B} \cdot\left(\mathbf{T}_{R G B}{ }^{t} \cdot \mathbf{T}_{R G B}\right)^{-1}
$$

It is well established that the basic color profile of an input device is a $3 \times 3$ matrix $\mathrm{M}$ (Eq. (1)) relating the colormatching functions of the color device $\mathbf{T}_{R G B}$ and the colorimetric standard observer $\mathbf{T}_{X Y Z}$. This means that the matrix components arranged in each column are the $X Y Z$ tristimulus values of the $R G B$ primaries of the input device (Eq. (4)). This matrix can be obtained by different regression methods. ${ }^{20}$ For this work, we have selected the maximum ignorance using least squares regression because it is the simplest procedure. Therefore, from these matrix components the color triangle of the input device can be plotted, as for displays and projectors, as a first approximation to the color gamut of this type of color device. That is, the maximum ignorance color transform is the projection of the primary fundamental spectra of the input device:

$$
\begin{aligned}
& \mathbf{M}=\left[\begin{array}{ccc}
X\left(\mathbf{P}_{R}\right) & X\left(\mathbf{P}_{R}\right) & X\left(\mathbf{P}_{B}\right) \\
Y\left(\mathbf{P}_{R}\right) & Y\left(\mathbf{P}_{G}\right) & Y\left(\mathbf{P}_{R}\right) \\
Z\left(\mathbf{P}_{R}\right) & Z\left(\mathbf{P}_{G}\right) & Z\left(\mathbf{P}_{B}\right)
\end{array}\right] \\
& \mathbf{M}=\mathbf{T}_{X Y Z}{ }^{t} \cdot \mathbf{T}_{R G B} \cdot\left(\mathbf{T}_{R G B}{ }^{t} \cdot \mathbf{T}_{R G B}\right)^{-1} \\
& =\left[\begin{array}{ccc}
1.5798 & 0.4016 & 0.3643 \\
1.0086 & 1.6157 & 0.0742 \\
-0.0107 & 0.1573 & 2.0189
\end{array}\right]
\end{aligned}
$$

where $\mathrm{P}_{R}, \mathbf{P}_{G}$ and $\mathbf{P}_{B}$ are the primary spectra in column format, which are not known a priori.

Since the matrix $\mathbf{M}$ obtained by the pseudo-inverse (or maximum ignorance) method does not fulfill the Luther condition, i.e., the CIE color matching functions estimated by $\mathbf{M} \cdot \mathbf{T}_{R G B}{ }^{\mathrm{T}}$ are not exactly equal to the original $\mathbf{T}_{X Y Z}$, we must actually talk of a family of metamers of the $R G B$ primaries for the camera, whose main representatives would be the primary fundamental spectra calculated above. The fact that they are $R G B$ metamers guarantees only that there will be a single $R G B$ triangle in the $(r, g)$ diagram of the camera, but this does not necessarily happen in the CIE chromaticity diagrams. Therefore, we may consider the color triangle of fundamental primaries as a first approxi- 
mation to the "camera alone" gamut. However, it would be very interesting to study how the possible families of $R G B$ primaries of the camera were encoded and plotted in the CIE color spaces.

A simple way to assess the color reproduction quality of an input device is to compare its colorimetric data- $-\mathbf{T}_{R G B}$, $\mathbf{P}_{R G B}$ and color triangle - with those of theoretical sensors or cameras verifying the Luther condition (Eq. (1)). As theoretical sensors, we might use either Ives-Abney-Yule's (Ref. 1, p 128) or MacAdam's22. In the first case, the color triangle of the sensor set is the minimal area that includes the spectral locus. In the second case, it is the exact linear combination of $\mathbf{T}_{X Y Z}$ with minimal spectral overlap. Obviously, none of these sensor sets exists in real input devices, but we think that it can be illustrative to use these theoretical data to analyze the color reproduction quality of current input devices.

Figure 1 shows the color-matching functions and primary fundamental spectra of our digital camera and the theoretical options of Ives-Abney-Yule and MacAdam. In Figure 2 the color triangles are compared in the $\left(u^{\prime}, v^{\prime}\right)$ chromaticity diagram. Since primaries might be nonreal, it must be born in mind that, when they are represented in a chromaticity diagram different from their own $(r, g)$, their additive mixtures will plot inside the triangle defined by the chromaticities of the primaries only if the sum of the tristimulus values of each primary in that representation space is positive. That is precisely the case of the primaries of our camera, even though their spectra have negative components and the tristimulus value $Z$ of the fundamental red primary is negative (see $\mathbf{M}$ in Eq. (4)). On the other hand, comparing the three color triangles, it can be seen that the red and purple region, and in a lesser degree also the blue-green region, are not well filled by our input device. We think that both the scaling of $\mathbf{T}_{R G B}$ relative to $\mathbf{T}_{X Y Z}$ and the spectral overlap in $\mathbf{T}_{R G B}$ are the cause of these differences. We have then a simple methodology to compare the color reproduction quality of cameras and scanners if their color-matching functions are known previously.

\section{Calculation of the Camera Responses to the Macadam Limits. Comparison with the CIE Standard Observer}

The camera responses of optimal colors can be obtained from the color-matching functions or optoelectronic spectral conversion functions of the camera. The input data are the spectral reflectances of the 1734 optimal colors under equalenergy illuminant $\mathrm{E}^{27}$. We consider that the illuminance of this hypothetic scene is $1000 \times 1$, obtained with a constant spectral radiance of $4.36 \mathrm{~mW} / \mathrm{sr} \mathrm{m}^{2}$, so the luminance of the adapting white is $L_{E}=1000 / \pi \mathrm{cd} / \mathrm{m}^{2}=318.31 \mathrm{~cd} / \mathrm{m}^{2}$. With these lighting data, the camera exposure was set to $N=4$ and $t=20 \mathrm{~ms}$ (offset value by manufacturer) and obtain the digital data $R G B$ of each optimal color using our previously developed model ${ }^{42,43}$.

From the camera responses, the $X Y Z$ tristimulus values of the optimal colors can be obtained applying any model of colorimetric characterization used. In our case, the digital data $R G B$ of each optimal color are the input of the reproduction model with luminance adaptation. The final step of our characterization model is to compare the estimated $X Y Z$ data with the theoretical $X Y Z$ data associated to the optimal colors. With this procedure we obtain the kind of color correction that our input device needs. As it is shown in Figure 3, a simple linear model (Eq. (5)), consisting of a scaling factor B and a Luther bias term A, works very well.

$$
\begin{aligned}
\mathbf{t}_{X Y Z}=\mathbf{A}+\mathbf{B} \cdot \hat{\mathbf{t}}_{X Y Z} & {\left[\begin{array}{c}
X \\
Y \\
Z
\end{array}\right]=\left[\begin{array}{l}
-19.02 \\
-24.57 \\
-21.44
\end{array}\right] } \\
+ & {\left[\begin{array}{ccc}
0.4705 & 0 & 0 \\
0 & 0.4320 & 0 \\
0 & 0 & 0.5436
\end{array}\right] \cdot\left[\begin{array}{c}
\hat{X} \\
\hat{Y} \\
\hat{Z}
\end{array}\right] }
\end{aligned}
$$

Nevertheless, it is important to remember that with Eq. (5) we compute a "camera plus transform" gamut, which aims to modeling as closely as possible the "camera alone" gamut. Of course, if the criteria ruling the projection over the $X Y Z$ color space changes, and therefore other matrix $\mathbf{M}$ is chosen, the color gamut would change. But, eventually, as a color correction model must be applied in the camera-tocolorimeter conversion, the choice of matrix $\mathbf{M}$ is not really too relevant. This is so because the main differences between both color gamuts are caused by the nonlinearities in the optoelectronic behavior of the digital camera and the nonfulfillment of the Luther condition.

This second approach is different from the procedure adopted by Morovič and Morovič's ${ }^{28}$. We use a "camera plus transform" to synthesize the camera's $R G B$ response to a set of theoretical reflectances (optimal or MacAdam colors) and then transform these $R G B$ values to $X Y Z$ space using a color transform. In this way a gamut is obtained in $X Y Z$ or another space defined in terms of $X Y Z$, e.g., $L^{*} a^{*} b^{*}$. Although such an approach is considered in Morovič and Morovič's work, it is rejected for a number of reasons. First, it is argued that to accurately determine the gamut of an input device one needs to sample the space of all possible surface reflectance functions. Thus, these authors aimed to determine the optimal color locus of their device, thus obtaining the "camera alone" gamut. A discussion of the computational problems associated with determining camera responses to all spectra in the object color solid (OCS) is given in that work, although the way their camera was modeled differs from ours. In addition, it is pointed out that a given $R G B$ response corresponds to a whole set of $X Y Z$ values due to the fact of metamerism (nonfulfillment of the Luther condition). This implies that the transformation from $R G B$ to $X Y Z$ is nontrivial, and in the case that a one-to-one correspondence is assumed, the transformation is subject to error. To avoid these problems they instead chose to define first the gamut of the OCS in $L^{*} a^{*} b^{*}$ space. However, we have simulated the $R G B$ responses for the optimal or MacAdam colors, which are on the boundary of the OCS, using our own characterization model $^{42}$ for digital cameras, 

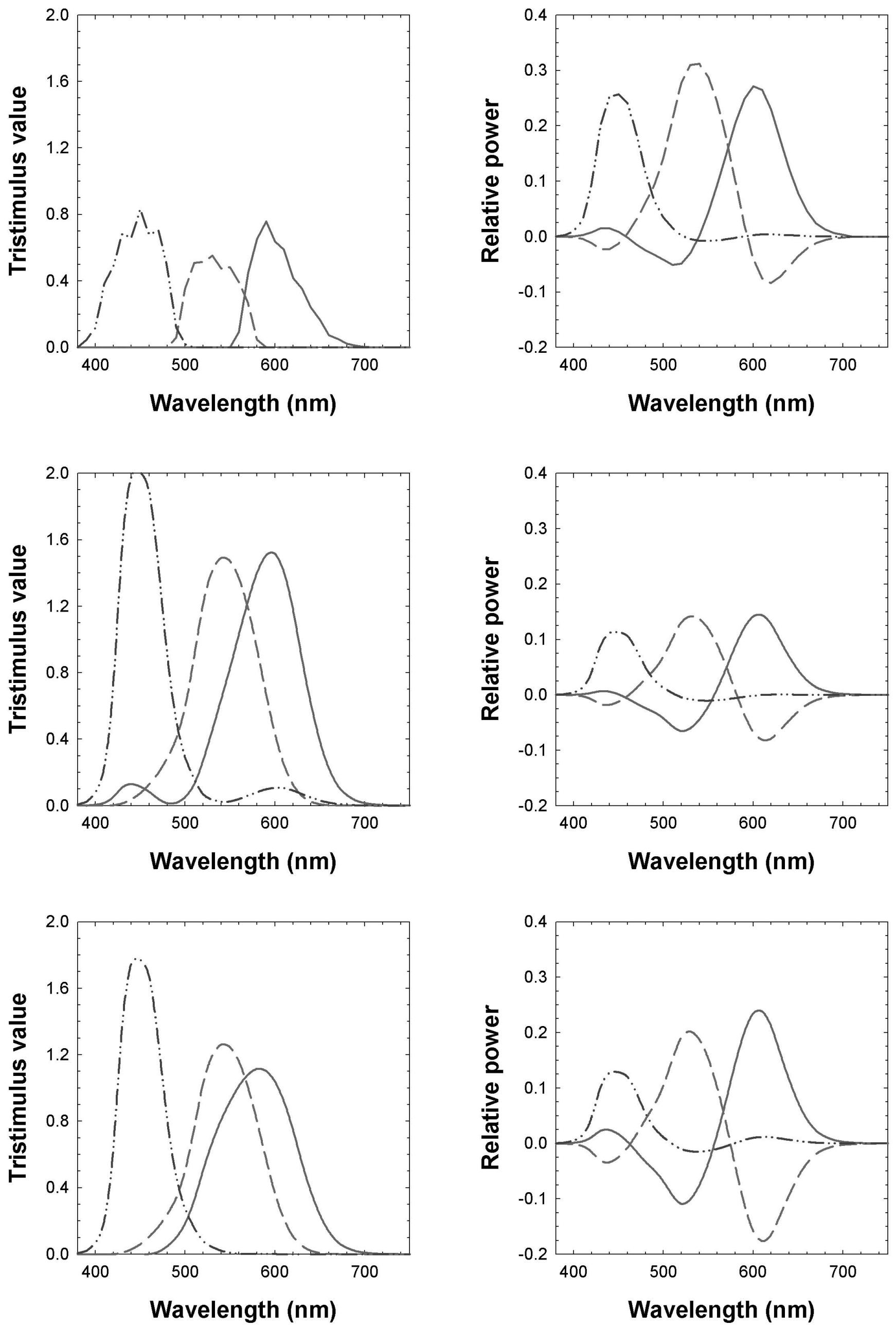


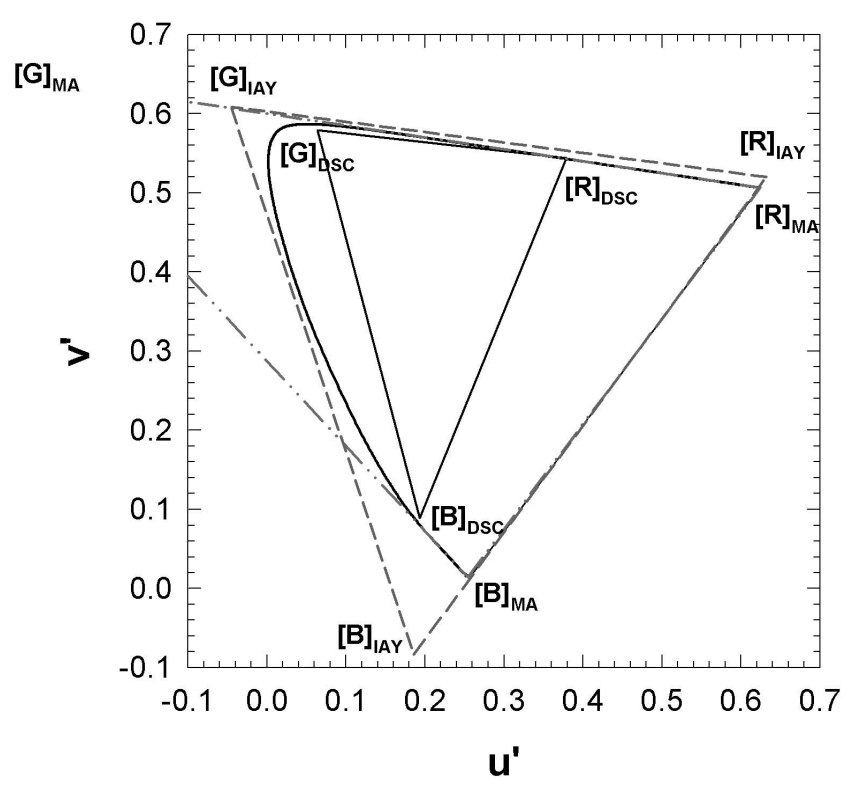

FIG. 2 Color triangles of our input device (DSC, solid line) and the theoretical options of Ives-Abney-Yule (IAY, dashed line) and MacAdam (MA, dash-dot-dotted line). [Color figure can be viewed in the online issue, which is available at www.interscience.wiley.com.]

mainly based on spectroradiometric measures. ${ }^{43}$ They then obtain an approximation of the camera gamut by sampling this $L^{*} a^{*} b^{*}$ space and determining camera $R G B$ values for each $L^{*} a^{*} b^{*}$ value sampled with a different camera model from that one of ours. In determining these camera $R G B$ values, the issue of metamerism is considered. That is, all reflectances corresponding to a given $L^{*} a^{*} b^{*}$ value are first determined. One of these reflectances is then selected and an $R G B$ value determined. In our case, we use an input device plus color transform to compute the camera response to the optimal colors of the human observer. But, as these colors, which are the borders of the OCS, have not got physically possible metamers (that is, with completely positive spectra), the problem of metamerism is minimized, in the sense that we need not be concerned by the possibility that a set of metamers of the colors used as reference might be nonmetameric for the camera and give a larger gamut. The remaining problem is that the algorithm used to predict the $X Y Z$ tristimulus values is affected by the metamerism of the camera. The two methods, therefore, are different approximations to the true solution.

Comparing the MacAdam limits in CIE- $\left(a^{*}, b^{*}\right)$ profiles, as shown in Figure 4, it is clear that the color gamut of the MacAdam colors encoded by our digital camera is smaller than that of the colorimetric standard observer. The same happens in $\left(a^{*}, L^{*}\right)$ and $\left(b^{*}, L^{*}\right)$ profiles in Figure 5, where it is clearly seen that our input device systematically losses more medium-light and dark colors than very light ones, and more colors in the red-green than in the yellow-blue chromatic axis.

Focusing only in the MacAdam limits encoded by the camera (Fig. 6), these seem much smaller when the luminance factor $Y$ is near 0 and 100 , that is, when very dark and light colors are captured. Taking into account the optoelectronic behavior of the camera, this seems logical because at very low and very high $Y$ values, the camera does not perform linearly. The camera exposure selected in this simulation seems optimal because the dynamic response range of the camera includes well the luminance ratio 100:1 of the simulated scene. The same happens if the exposure is slightly increased up to 5.6, without changing the lighting conditions of the virtual scene. But in other exposure configurations where the color profile was kept completely linear, either by overexposure ( $N<5.6, t$ fixed) or underexposure $(N>5.6, t$ fixed), the color gamut of our input device changes and decreases noticeably (Fig. 6). In this last figure, it can be seen how with a smaller camera exposure $(N=8)$, the gamut of dark colors is greatly decreased in comparison with $N=5.6$, at the expense of maintaining approximately the gamut of the very light colors. On the other hand, with a bigger camera exposure $(N=3.7)$, although the camera saturates very light colors $(Y>80)$, the gamut of light and dark colors does not change greatly. Therefore, if one has to choose an incorrect camera exposure, it is better to overexpose (saturating the white target of the scene, as in conventional photographic practice) than to underexpose.

Another topic that should be taken into account is the possibility of having in the scene a luminance ratio larger than 100:1, as for example 512:1 in other capture conditions. ${ }^{45,46}$ In such case, we could vary the f-number $N$ of the zoom-lens to provide such luminance adaptation as our color profile allows. Obviously, the optoelectronic (OECSF) and photometric properties of our camera influence the optimal capture for luminance ratios equal to 100:1, typical of many scenes. But it is possible that in other input devices, with a shorter dynamic response range, the darker or lighter colors would be clipped independently of the selected exposure configuration. Therefore, our methodology can be used to compare the color reproduction capabilities of input devices.

\section{Concerning the Reduction of the Distinguishable Colors by the Camera}

A simple way of estimating the size of the color gamuts of a device is to plot a reference color locus, transformed by the device, in some color diagram, for instance CIE$L^{*} a^{*} b^{*}$, and to assume that the area of this locus is a

FIG. 1 Color-matching functions (left side) and fundamental primary spectra (right side) of our digital camera (top) and the theoretical cameras of Ives-Abney-Yule (center) and MacAdam (bottom). Solid line, R channel; dashed line, G channel; dash-dot-dotted line, B channel. [Color figure can be viewed in the online issue, which is available at www.interscience. wiley.com.] 

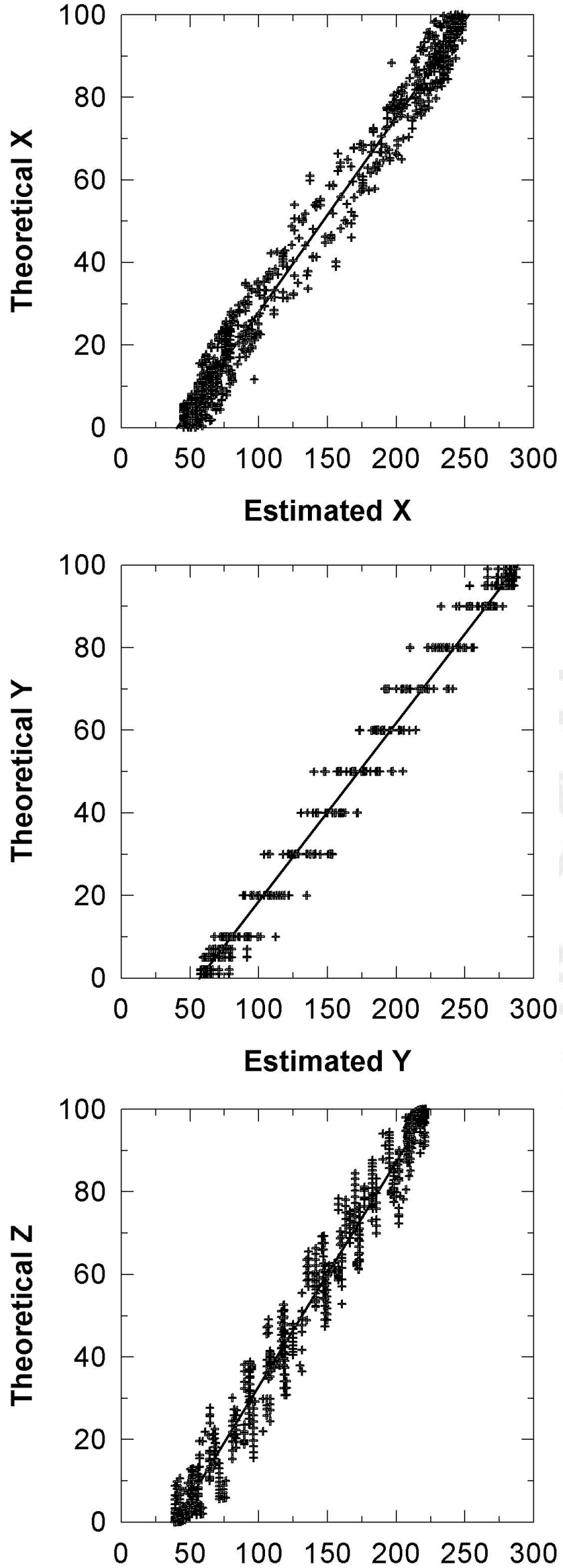

Estimated Z
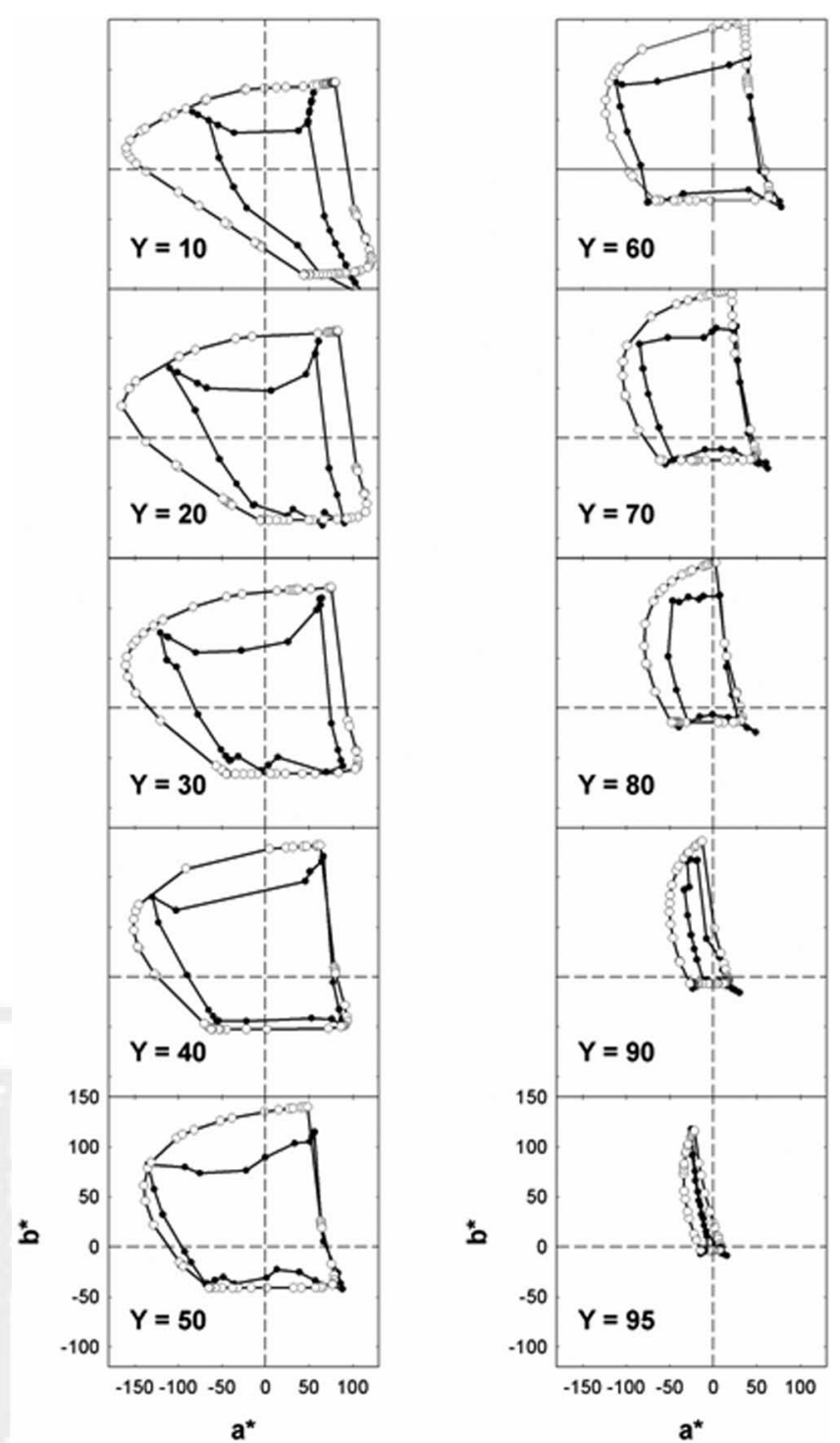

FIG. 4 MacAdam limits with different luminance factors in the CIE- $\left(a^{*}, b^{\star}\right)$ diagram, for the CIE-XYZ standard observer (outer line with hollow symbols) and for our digital camera (inner lines with solid symbols).

measurement of the gamut size. With this technique, we are choosing to ignore the fact that this space is not really uniform. This could seem a reasonable simplification to obtain a first order approximation, but as we show in Figure 7, the consequences of this fact can be serious: the conclusions depend not only on the device under analysis but also, and strongly, on the representation space used, even for those with reasonable uniformity. The scaling changes, but the more relevant features are the changes in the shapes of the curves, resulting in the prediction of different relative decrements in the gamut for different luminance factors in the different color spaces used.

FIG. 3 Linear correction (solid line) of the tristimulus values $X$ (top), $Y$ (center), and $Z$ (bottom) of 1734 optimal colors (crosshair symbol) using the color profile associated to our input device. The correlation indexes are 0.9892, 0.9952, and 0.9844 , respectively. 

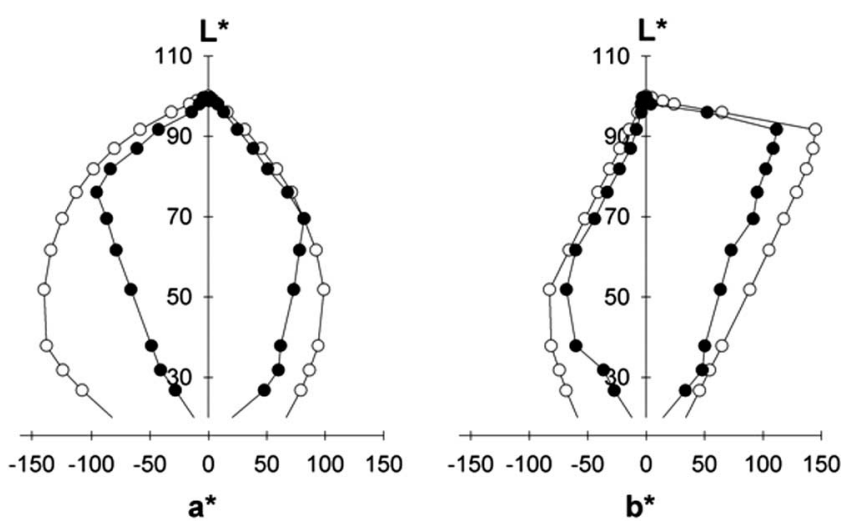

FIG. 5 Profiles $a^{*}$ versus $L^{*}$ (left) and $b^{*}$ versus $L^{*}$ (right) of the Rösch-MacAdam color solid under equal-energy illuminant for the colorimetric standard observer (line with hollow symbols) and encoded by our digital camera (line with solid symbols) with exposure configuration $N=4$ and $t=20 \mathrm{~ms}$.

A possible solution would be to work with the most uniform of the available color spaces. We have chosen, however, a different approach consisting in counting the number of distinguishable colors within the reference locus - in the occurrence, the optimal color loci. This has been done by computing the discrimination ellipses with a discrimination model derived from the experimental data of Krauskopf and Gegenfurtner. ${ }^{37,38}$ This is done in a modified MacLeod-Boynton color space, ${ }^{47}$ where the cromaticity coordinates for the equal-energy, perceptual, or adapted white stimulus were $(0,0)$. The new cromaticity coordinates $\left(l^{\prime}, s^{\prime}\right)$ are $l^{\prime}=l-l_{E}$ and $s^{\prime}=s-s_{E}$ where $l_{E}=0.66537$ and $s_{E}$ $=0.01608$, after calculating the cone excitations LMS of the white stimulus $E\left(X_{E}=Y_{E}=Z_{E}=100\right)$ using the Smith-Pokorny fundamental matrix. These new chromaticity coordinates are rescaled in such a way that the threshold around the equal energy white along the cardinal directions is one, that is, $l^{\prime}$ and $s^{\prime}$ are divided by, respectively 0.0011 and 0.0012 , according to the Krauskopf and Gegenfurtner data. At constant luminance (constant $L+M$ ) the response of any red-green opponent mechanism, of the form $T=L-$ $\alpha M$, depends linearly on the scaled $l^{\prime}$ coordinate, and any blue-yellow mechanism, of the form $D=S-\beta(L+M)$, would change linearly with the scaled $s^{\prime}$ coordinate. For this reason, although in the next figures we plot $s^{\prime} / 0.0012$ versus $l^{\prime} / 0.0011$, we use the labels $S-(L+M)$ (short for $D=S$ $-\beta(L+M)$ ) and $L-M$ (which in the literature is often used as short for $T=L-\alpha M$ ) for the vertical and horizontal axes, respectively. In this color space, colors in the same vertical line in the chromatic diagram have constant $L$ and $M$ values, while colors in the same horizontal line have constant values for $S$ and $(L+M)$. Accordingly, a vertical line contains colors that would give constant response in a red-green mechanism, $T=L-\alpha M$, no matter the value of $\alpha$. Analogously, a horizontal line contains colors yielding constant response in a yellow-blue mechanism of the type $D=S-\beta(L+M)$, no matter the value of $\beta$. In particular, those colors in the $D=0$ and $T=0$ lines elicit responses only from the $T$ or the $D$ mechanism, respectively, and are therefore the cardinal directions of $T$ and $D$.

In this color space, the discrimination ellipses are computed as follows. The discrimination ellipse around the equal-energy white $(T=0, D=0)$ defines the unity threshold in each cardinal direction. Thus, with this metric the discrimination ellipse around $(T=0, D=0)$ is a circle of unity radius. Let us consider a pedestal in the $T$ cardinal direction. Thresholds along this direction are proportional to the $T$ response to the pedestal, whereas thresholds along the orthogonal $D$ direction are constant. Analogously, if the pedestal is on cardinal direction $D$, thresholds along the $D$ direction are proportional to the $D$ response to the pedestal, whereas they are constant along the orthogonal $T$ direction
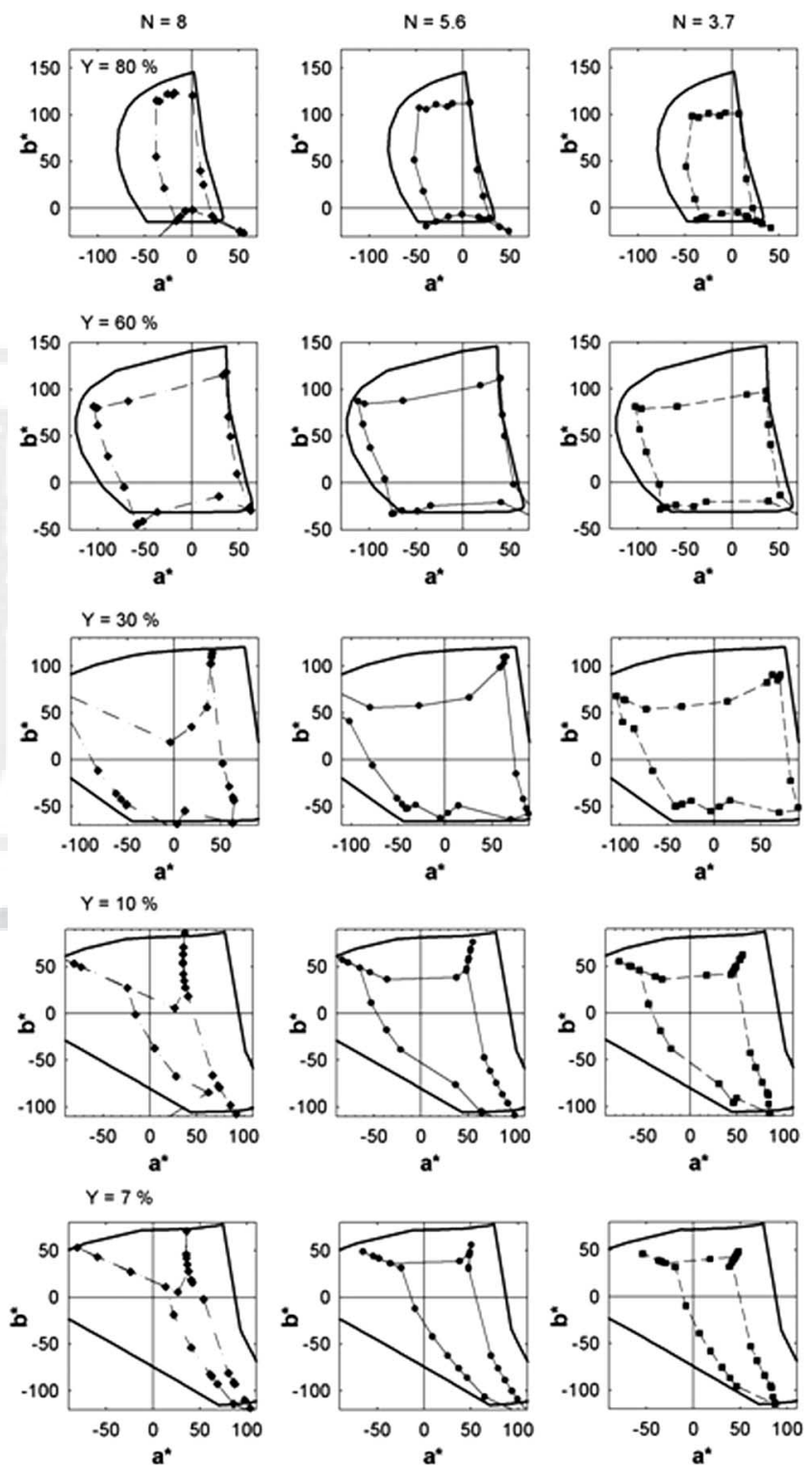

FIG. 6 Responses of the camera to the MacAdam loci compared with the original stimuli, for different camera exposures. From bottom to top: data for luminance factor $Y=$ $\{7,10,30,60,80\}$, that is, from darker to lighter colors. From left to right side: camera exposures $N=\{8,5.6,3.7\}$ with $t=$ $20 \mathrm{~ms}$, that is, from smaller to bigger exposure. 


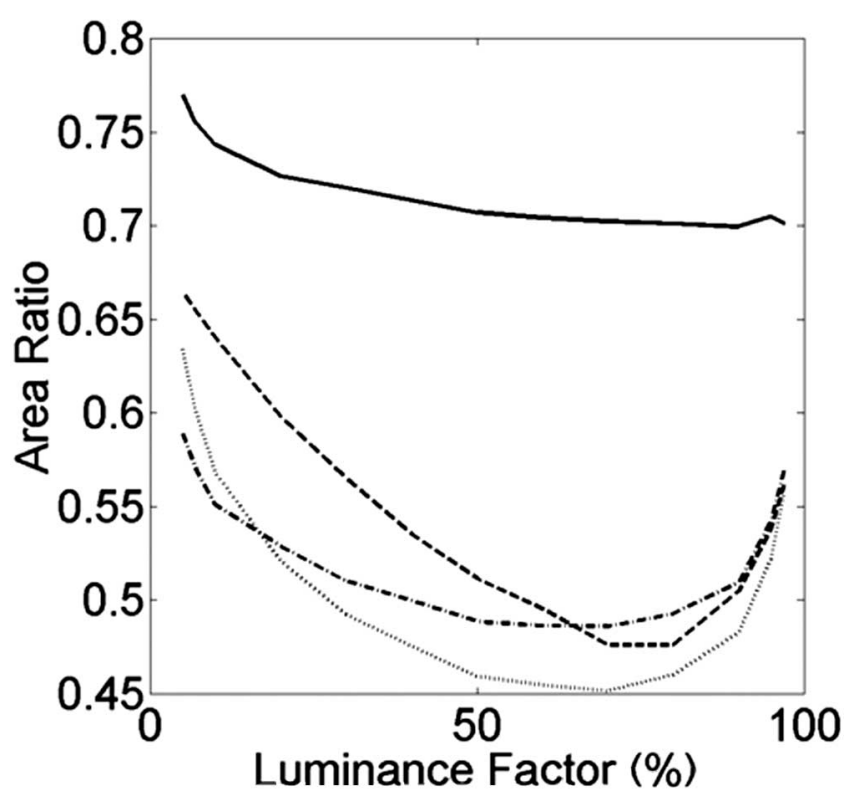

FIG. 7 Influence of the choice of the color space on the conclusions about the color gamut of device derived from its area in a chromaticity diagram. To illustrate the problem, we have considered a theoretical camera, whose only effect on the colors of the scene is a reduction in colorimetric purity by a constant factor (0.8). The responses to the MacAdam loci at different luminance factor $Y_{S}(\%)$ were computed. The area of the resulting loci was then divided by the area of the original MacAdam loci. Although all the spaces used are reasonably uniform (the most uniform one being SVF), the numerical values and the overall shape of the curves differ widely. Results in CIELAB (dotted line), SVF (dashed line), RLAB (dash-dotted line), and LLAB (with a zero luminance background) (solid line).

(Fig. 8). In consequence, discrimination ellipses around stimuli in one of the cardinal directions are oriented along that direction. The rate at which the major axis of each ellipse changes along each cardinal direction was taken from the experimental data of Krauskopf and Gegenfurtner. When the pedestal is not on one of the cardinal directions, the laws governing thresholds are not so simple. Discrimination ellipses around a pedestal in the first or third quadrant of the modified MacLeod-Boynton space seem to be oriented along the cardinal directions. The sizes of the major and minor axis of the ellipses are proportional to the $T$ or $D$ response elicited by the pedestal. This result can be explained if we admit the existence of two independent discrimination mechanisms, whose cardinal directions are the $T$ and $D$ directions of MacLeod-Boynton's diagram, and that interact vectorially. However, discrimination ellipses around pedestals in the second or fourth quadrant seem to be oriented along the direction defined by the pedestal. This result seems to imply the existence of a continuum of mechanisms tuned along equally spaced directions in the color space. The directions along which are tuned these hypothetical mechanisms could be deduced approximately from the experimental data, but the rate of increment of threshold along each of these directions cannot. Although it could reasonably be admitted that thresholds again would increase with increasing distance to the white stimulus, the actual law of variation would still to be determined. Because our aim is to compare the number of ellipses within the MacAdan limits in the human observer and the camera, and not to reach the best estimation of this number, the model of two cardinal directions is enough. To avoid further complications, we will assume that the variation laws of thresholds we have described for a constant luminance plane are independent from luminance, which is reasonably true ${ }^{48}$ at least down to $1 \mathrm{~cd} / \mathrm{m}^{2}$.

The next problem to solve is which method to use to pack the discrimination ellipses. We have followed two different procedures. With what we call the tangent criterion, we determine the position of the centers of the ellipses to verify two conditions: (1) each ellipse is tangent to the other four surrounding it and (2) the centers of two adjacent ellipses have either the same $T$ or the same $D$ value. This criterion does not yield optimal packing, because the gaps between ellipses increase with the distance to the achromatic
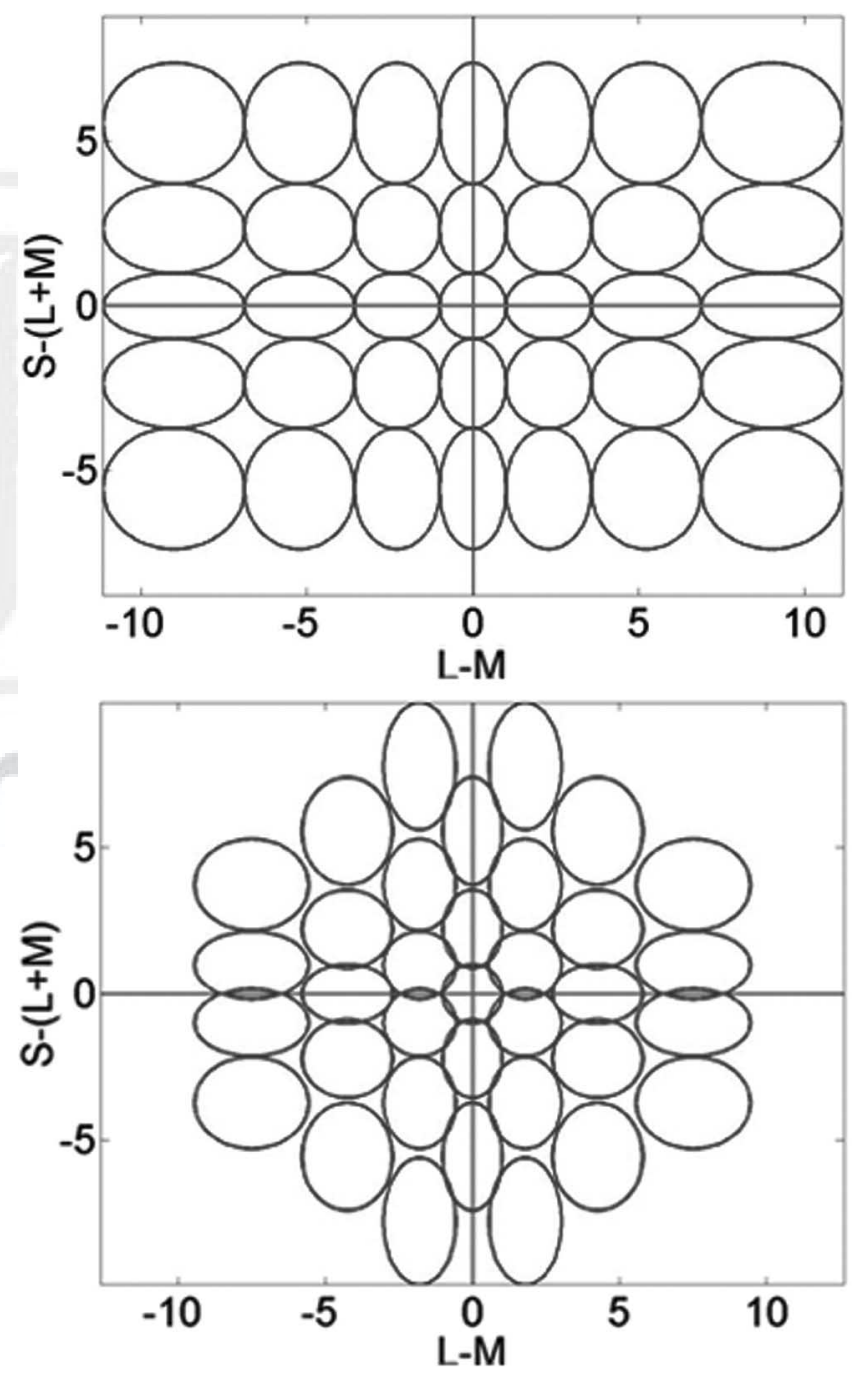

FIG. 8 Example of tangent (top) and dense (bottom) arrangement of discrimination ellipses in a constant luminance plane when two independent mechanisms ( $T$ and $D)$ determine threshold. [Color figure can be viewed in the online issue, which is available at www.interscience.wiley.com.] 

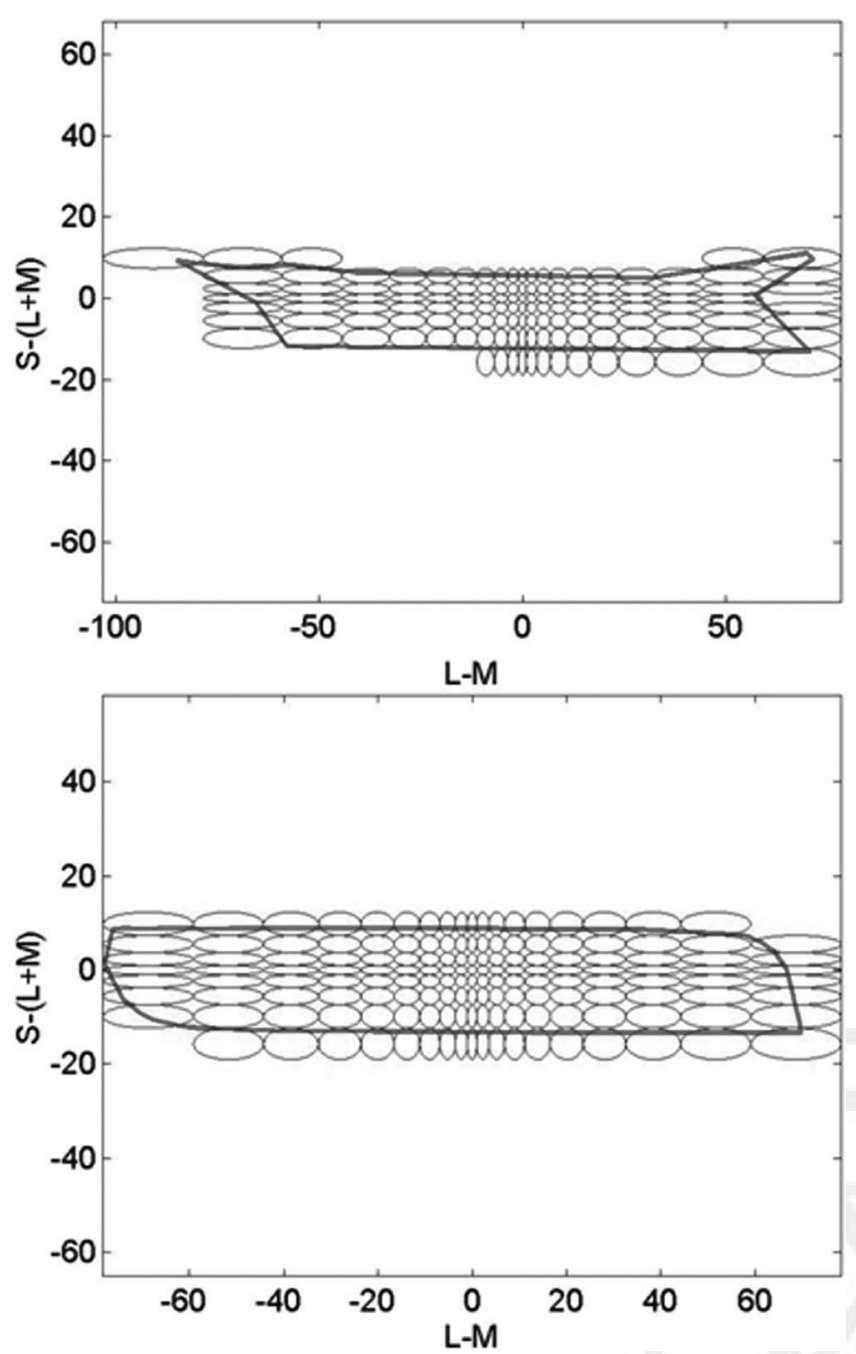

FIG. 9 Tangent packing of the discrimination ellipses inside the MacAdam limits for the luminance factor $Y=60 \%$ for the digital camera (top), with exposure configuration $N=4$ and $t=20 \mathrm{~ms}$, and for the human eye (bottom). The number of discernible colors corresponding to each case is 131 and 150 , respectively. [Color figure can be viewed in the online issue, which is available at www.interscience.wiley.com.]

point. ${ }^{49,50}$ The second strategy, that we call dense packing, consists in placing the centers of the ellipses on the centers of the tiles of an hexagonal mosaic covering optimally the space to which we have applied a nonlinear transform $\left[x^{*} f(x), y^{*} f(y)\right]$. The functions $f(x)$ and $f(y)$ have been found empirically, and verify that the overlap between ellipses is small. In this way, we come nearer to optimum ellipse packing (Fig. 8). The results obtained for the human eye and our camera for $Y=60 \%$ and the tangent criterion are shown as an example in Figure 9. In Figure 10 we show the results derived from the dense packing criterion.

The two packing criteria produce basically the same results, as can be seen in Figure 11, where we have plotted the number of distinguishable colors versus the luminance factor, both for the human eye and our camera. In the range of luminance factors explored, which goes as low as 5\%, both curves associated to our digital camera have the same behavior relative to the human eye: as expected, the number of distinguishable colors for the camera is lower than for of the human eye. Obviously, we would expect that the number of distinguishable colors for the standard observer (and also the camera) would decrease as the luminance factor approaches to zero (ideal black). With the discrimination model used, the number of ellipses would increase indefinitely as the luminance factor decreases. Let us remember, however, that our model did not include the influence of the adapting luminance on discrimination thresholds. Therefore, the model should not be used for low luminances and we must admit some uncertainty about the luminance factor below which the number of discriminable colors decreases.

Figure 12 also shows some interesting features. Here we show the change in the number of colors than can be distinguished by the camera (with several exposure configurations) relative to the standard colorimetric observer, as a function of lightness. In the middle range of $L^{*}$ values, the relative reduction factor is approximately constant, particu-
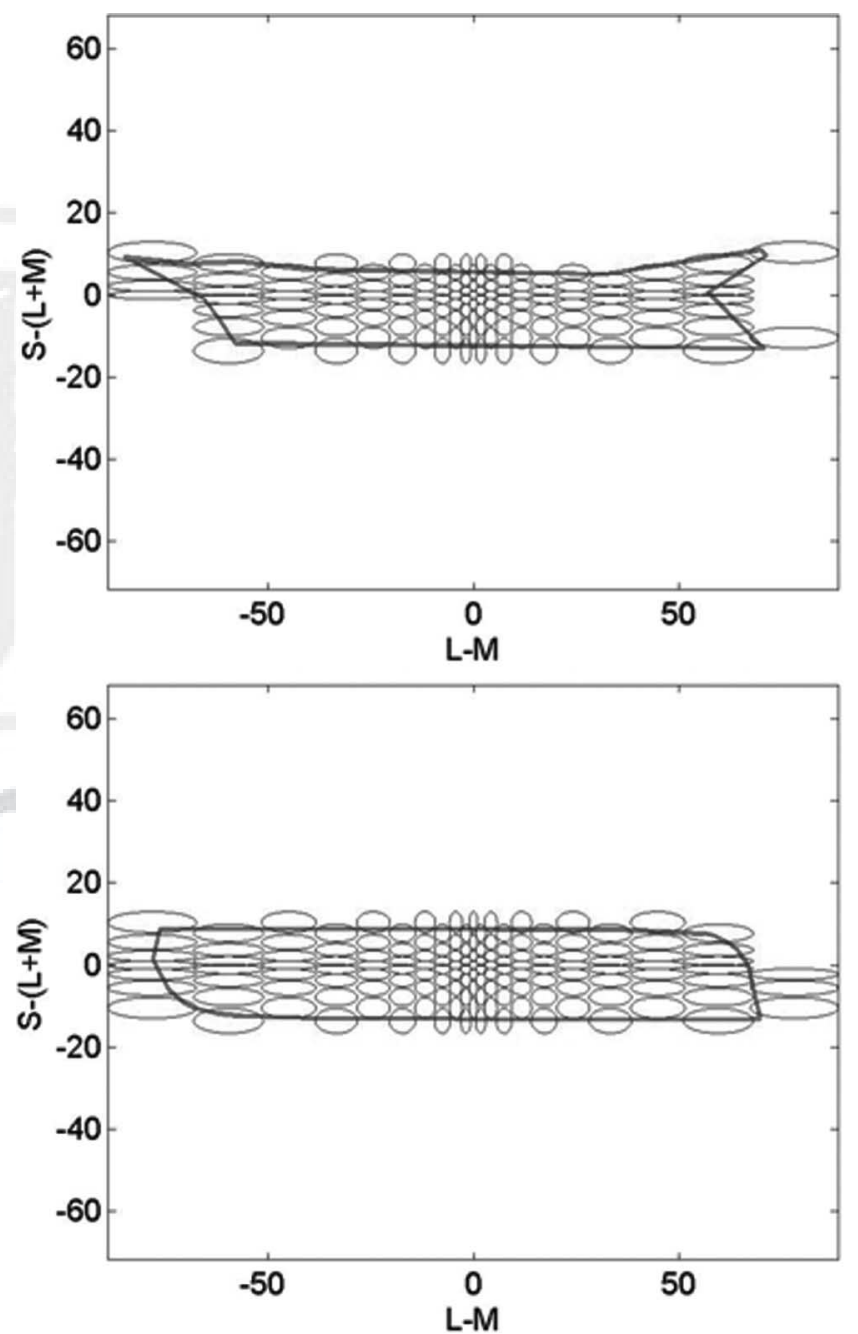

FIG. 10 Dense packing of the discrimination ellipses inside the MacAdam limits for the luminance factor $Y=60 \%$ for the digital camera (top), with exposure configuration $N=4$ and $t=20 \mathrm{~ms}$, and for the human eye (bottom). The number of discernible colors corresponding to each case is 128 and 143 , respectively. [Color figure can be viewed in the online issue, which is available at www.interscience.wiley.com.] 


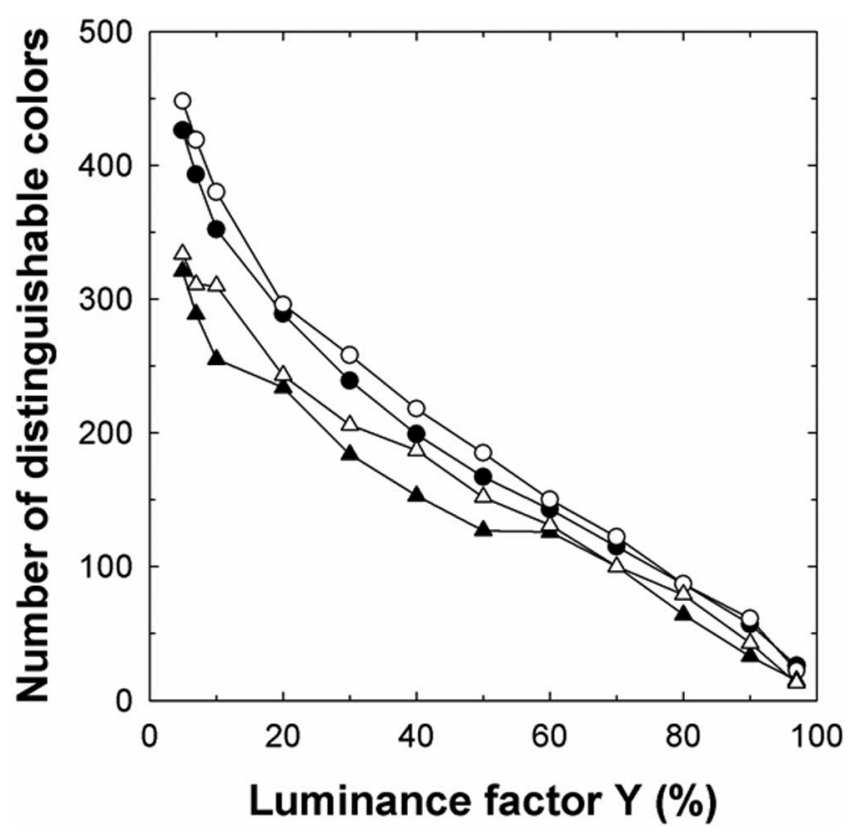

FIG. 11 Number of discernible colors according to the luminance factor and ellipses packing method (dense, solid symbol; tangent, hollow symbol) of the discrimination ellipses in the digital camera (triangle) and the human eye (circle). The camera exposure is $N=4$ and $t=20 \mathrm{~ms}$.

larly for $N=4$ and 5.6, with a slightly depression or absolute minimum around $L^{*}=80(Y=60 \%)$. That is, with this exposure value, the camera distinguishes a lesser number of very dark than of very light colors and a relatively larger number of colors with intermediate lightness ( $Y$ between 40 and 70, or $L^{*}$ between 69.5 and 87.0). Then, this suggests that the linear range of the digital camera is similar for these two $N$ values when the overall illumination level of the scene is kept constant. When the camera is overexposed (i.e. $N=3.7$ ), the relative decrement of distinguishable colors is higher, but the trends in the curve are similar to those obtained for $N=4$ and 5.6. The same thing happens when the camera is underexposed (i.e. $N=8$ ), but particularly the relative decrement of distinguishable colors is higher for dark than for very light colors. Therefore, as we advanced above, it is better to overexpose than to underexpose. Both behaviors happen because the dynamic range of the digital camera is shorter than that of the human eye: the number of distinguishable dark and light colors decreases compared with the human eye because the camera response near the regions where, respectively, noise and saturation impair performance. Nevertheless, both behaviors are not symmetrical, above all in the relative decrement of the dark colors.

\section{CONCLUSIONS}

The knowledge of the color gamut of an input device is important in Color Imaging but its determination is complex, because these color devices verify the univariance principle but do not fulfill the Luther condition. Moreover, optoelectronic performance is not completely ideal for the basic assumptions of colorimetry to hold. We have presented in this work some methods to reach an acceptable solution of this problem, which can be applied to any digital camera or scanner.

The first procedure we have developed is based on the determination of the color triangle of these color devices from the fundamental primary spectra related to the "camera alone" gamut. Although this algorithm is simple, it is very useful because we use theoretical sensors (Ives-Abney-Yule and MacAdam) as reference. In this way, we can determine the effect of the scaling of the spectral sensitivities and its spectral overlap on the shape of the color triangle.

The second procedure is based on the simulated capture and encoding of the optimal or MacAdam colors under equal-energy illuminant and its comparison relative to the chromatic encoding by the human eye (CIE-1931 XYZ). We have shown that, in its raw performance and using the optimal exposure configurations $(N=4$ or $5.6, t=20 \mathrm{~ms})$, our digital camera has smaller MacAdam loci than the human eye. If the camera exposure varies from this optimal exposure value, either by overexposure $(N<4)$ or by underexposure $(N>5.6)$, the color gamut is different, above all in the darker colors, respectively. Therefore, this algorithm can be useful to compare the color gamut areas of digital cameras and scanners. With scanners, this analysis is limited because it is not possible to change at hardware level the manufacturer camera exposure (or the f-number $N$ or the exposure time $t$ ). However, this approach is basically different to the Morovič and Morovič's work, ${ }^{28}$ although both

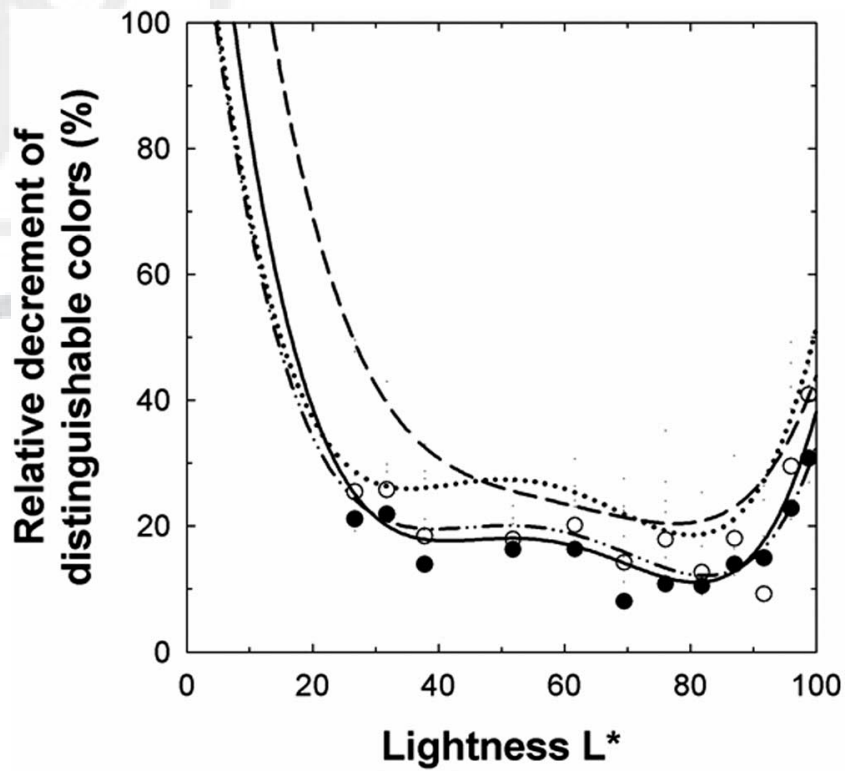

FIG. 12 Relative decrement of the discernible colors versus lightness with several exposure configurations (solid line, $N=4$; dashed line, $N=8$; dotted line, $N=3.7$; dash-dot-dot line, $N=5.6)$. The plotted symbols correspond to camera exposure $N=4$ and $t=20 \mathrm{~ms}$ (solid symbols, dense packing; hollow symbols, tangent packing). The plotted lines correspond to the fourth-order polynomial fitting curves for each data group combining the dense and tangent packing data. 
methods can give similar results using the same input device and its color model, because they try to solve the problem of the color gamut of an input device calculating the "camera alone" gamut taking into account the device metamerism. On other hand, we try to solve this problem from the "camera plus transform" gamut approach taking into account the human optimal colors. Thus, one can choose one or other method depending on the algorithmic complexity and computational time to reach the solution, but always in both methods an approximate solution. Therefore, we think that the correct approach solving this problem should be for a near future to calculate the optimal or MacAdam colors associated to the input device and compare this color gamut with those of the human eye in CIE (human) color spaces.

Supplementary to this second approach to define the problem of the color gamut of input devices, the number of discernible colors by our digital camera and human eye has been estimated and compared. To do this we assume that our input device has the same color metric as the human eye, although this implies nothing about device metamerism. Thresholds have been computed with the chromatic discrimination model of Krauskopf and Gegenfurtner, in a modified MacLeod-Boynton chromatic diagram, using two packing methods, tangent and dense, reaching similar results with both cases. The central point of our analysis, in relation with the nonideal optoelectronic and colorimetric performance of the analyzed input device, is that depending on the camera exposure there is a lightness range where the relative decrement of the distinguishable colors by the camera relative to the human eye is approximately constant. Under optimal exposure conditions $(N=4$ or 5.6 and $t=20$ $\mathrm{ms}$ for $E=1000 \times 1$ ), our digital camera distinguishes considerably fewer very dark colors than very light ones, but relatively much more colors with middle lightness ( $Y$ between 40 and 70 , or $L^{*}$ between 69.5 and 87.0). Therefore, as before, this last procedure can also be used to compare the color reproduction quality of digital cameras and scanners.

\section{ACKNOWLEDGMENTS}

We wish to thank Prof. JL Monterde García-Pozuelo, member of the Department of Geometry and Topology of the University of Valencia, for valuable advice on packing algorithms with ellipses. The authors would like to thank the referees for their advices, helpful comments, and suggestions.

1. Hunt RWG. The Reproduction of Colour, 5th edition. Kingston-uponThames: Fountain Press; 1995.

2. Sharma G, Trussell HJ. Digital color imaging. IEEE Trans Image Process 1997;7:901-932.

3. Kang HR. Color Technology for Electronic Devices. Bellingham: SPIE Press; 1997.

4. Green P. Understanding Digital Color, 2nd edition. Pittsburgh: GATF Press; 1999.

5. Green P, MacDonald LW. Colour Engineering: Achieving Device Independent Colour. Chichester: Wiley; 2002.

6. MacDonald LW, Luo MR, Colour Image Science: Exploiting Digital Media. Chichester: Wiley; 2002.
7. Wandell, BA, Silverstein DL. Digital color reproduction. In: Shevell SK, editor. Science of Color, 2nd edition. New York: Elsevier; 2003. pp 281-316.

8. Kipphan H. Handbook of Print Media: Technologies and Production Methods. Berlin: Springer; 2001.

9. Giorgianni EJ, Madden TE. Digital Color Management: Encoding Solutions. Reading: Addison-Wesley; 1998.

10. Adams RM, Weisberg JB. The GATF Practical Guide to Color Management, 2nd edition. Pittsburgh: GATF Press; 2000.

11. International Color Consortium. File Format for Color Profiles (Version 4), Peston: International Color Consortium; 2001. Specification ICC. 1:2001-12. Available at www.color.org.

12. Morovič J, Sun PL. How different are colour gamuts in cross-media colour reproduction? In: MacDonald LW, Luo MR, editors. Colour Image Science. Chichester: Wiley; 2002. pp 237-258.

13. Mahy M. Colour gamut determination. In: Green P, MacDonald LW, editors. Colour Engineering. Chichester: Wiley; 2002. pp 263-295.

14. Joshi AM, Olsen GH. Photodetection. In: Bass M, editor. Handbook of Optics, Vol. I, 2nd edition. New York: McGraw-Hill; 1995. pp 16.116.21 .

15. Tredwell TJ. Visible array detectors. In: Bass M, editor. Handbook of Optics, 2nd edition. New York: McGraw-Hill; 1995. Vol. 1, pp 22.122.38.

16. Holst GC. CCD Arrays, Cameras and Displays, 2nd edition. Bellingham: SPIE Press; 1998

17. Luther R. Aus dem gebiel der farbreizmetrik. Z Tech Phys 1927;8: $540-558$.

18. Horn BKP. Exact reproduction of colored images. Comput Vis Graph Image Process 1984;26:135-167.

19. Brainard DH. Colorimetry. In: Bass M, editor. Handbook of Optics, 2nd edition. New York: McGraw-Hill; 1995. Vol. 1, pp 26.1-26.54.

20. Finlayson GD, Drew MS. Constrained least-squares regression in colour spaces. J Electron Imaging 1997;6:484-493.

21. Vrhel MJ, Trussell HJ. Color device calibration: A mathematical formulation. IEEE Trans Image Process 1999;8:1796-1806.

22. Pearson ML, Yule AC. Transformation of color mixture functions without negative Portions. J Color Appearance 1973;2:30-35.

23. Kotera $\mathrm{H}$. Generation of virtual spectral color target and application to testing input devices. J Imaging Sci Technol 2001;45:373-383.

24. Rommey AK, Indow T. Munsell reflectance spectra represented in three-dimensional euclidean space. Color Res Appl 2003;28:182-196.

25. MacAdam DL. Maximum visual efficiency of colored materials. In: MacAdam DL, editor. Selected Papers on Colorimetry. Bellingham: SPIE Press; 1993.pp 244-250.

26. Wyszecki G, Stiles WS. Color Science: Concepts and Methods, Quantitative Data and Formulae, 2nd edition. New York: Wiley; 1982; pp 179-183.

27. Perales E, Mora T, Viqueira V, de Fez D, Gilabert E, Martínez-Verdú F. A new algorithm for calculating the MacAdam limits for any luminance factor, hue angle and illuminant. In: Tenth Congress of the International Colour Association, Granada, 2005. pp 737-740.

28. Morovič J, Morovič P. Determining colour gamuts of digital cameras and scanners. Color Res Appl 2003;28:59-68.

29. Berns RS. Billmeyer and Saltzman's Principles of Color Technology, 3rd edition. New York: Wiley; 2000; p 62, 143.

30. Pointer MR, Attridge GG. The number of discernible colours. Color Res Appl 1998;23:52-54.

31. Kuehni, RG. Color Space and Its Divisions: Color Order from Antiquity to the Present. New York: Wiley; 2003. p 202.

32. MacAdam DL, Visual sensitivities to color differences in daylight. J Opt Soc Am 1942;32:247-274.

33. Brown WRJ, MacAdam DL. Visual sensitivities to combined chromaticity and luminance differences. J Opt Soc Am 1949;39:808-834.

34. Wyszecki G, Fielder GH. New color-matching ellipses. J Opt Soc Am 1971;61:1135-1152.

35. Romero J, García JA, Jiménez del Barco L, Hita E. Evaluation of color-discrimination ellipsoids in two color spaces. J Opt Soc Am A 1993;10:827-837. 
36. Carreño F, Zoido JM. The influence of luminance on color-difference thresholds. Color Res Appl 2001;26:362-368.

37. Krauskopf J, Gegenfurtner KR. Color discrimination and adaptation. Vis Res 1992;32:2165-2175.

38. Krauskopf J. Higher order color mechanisms. In: Gegenfurtner KR, Sharpe LT, editors. Color Vision: From Genes to Perception. Cambridge: Cambridge University Press; 1999. p 310.

39. Photography-Electronic Still Picture Cameras-Methods for Measuring Opto-Electronic Conversion Functions (OECFs), ISO 14524, Geneva: ISO; 1999. Available at www.iso.ch.

40. Graphic Technology and Photography-Colour Characterization of Digital Still Cameras (DSCs), ISO 17321:2002, Geneva: ISO; 2002. Available at www.iso.ch.

41. Balasubramanian R. Device characterization. In: Sharma G, editor. Digital Color Imaging Handbook. Boca Raton: CRC Press; 2003. Chapter 5.

42. Martínez-Verdú F, Pujol J, Capilla P. Characterization of a digital camera as an absolute tristimulus colorimeter. J Imaging Sci Technol 2003;47:279-295.
43. Martínez-Verdú F, Pujol J, Capilla P. Calculation of the color matching functions of digital cameras from their complete spectral sensitivities. J Imaging Sci Technol 2002;46:15-25.

44. Cohen JB. Color and color mixture: Scalar and vector fundamentals. Color Res Appl 1988;13:5-39.

45. Jacobson RE, Attridge GG, Ray SF, Axford NR. The Manual of Photography. Photographic and digital imaging, 9th edition. Oxford: Focal Press; 2000.

46. Stroebel L, Compton J, Current I, Zakia R. Basic Photographic Materials and Processes, 2nd edition. Boston: Focal Press; 2000.

47. MacLeod DI, Boynton RM. Chromaticity diagram showing cone excitation by stimuli of equal luminance. J Opt Soc Am 1979;69:11831186.

48. Brown WRJ. The influence of luminance level on visual sensitivity to color differences. J Opt Soc Am 1951;41:684-688.

49. Berns RS. Billmeyer and Saltzman's Principles of Color Technology, 3rd edition. New York: Wiley; 2000. pp 115-121.

50. Luo MR, Cui G, Rigg B. The development of the CIE 2000 colourdifference formula: CIEDE2000. Color Res Appl 2001;26:340-350. 